EPJ Web of Conferences 113,04002 (2016)

DOI: $10.1051 /$ epjconf/201611304002

C Owned by the authors, published by EDP Sciences, 2016

\title{
Studies of three-nucleon systems with improved chiral forces
}

\author{
R.Skibiński ${ }^{1}$,, J.Golak ${ }^{1}$, K.Topolnicki ${ }^{1}$, H.Witała ${ }^{1}$, and E.Epelbaum ${ }^{2}$ \\ ${ }^{1}$ M.Smoluchowski Institute of Physics, Jagiellonian University, PL-30348 Kraków, Poland \\ ${ }^{2}$ Institut für Theoretische Physik II, Ruhr-Universität Bochum, D-44780 Bochum, Germany
}

\begin{abstract}
Recently developed chiral two-nucleon (2N) potentials are applied to various processes in three-nucleon $(3 \mathrm{~N})$ systems within the approach based on Faddeev equations. The predictions for the cross sections in elastic nucleon-deuteron $(\mathrm{Nd})$ scattering and for radiative proton-deuteron (pd) capture are similar to results based on the AV18 potential. The very good convergence with respect to the chiral expansion and a weak dependence on a regularization parameter are observed. This gives hope that the Hamiltonian combining these $2 \mathrm{~N}$ potentials with consistent $3 \mathrm{~N}$ forces will become an important tool to study nuclear structure and processes.
\end{abstract}

\section{Introduction}

Recently, the improved $2 \mathrm{~N}$ forces up to next-to-next-to-next-to-next-to leading order $\left(\mathrm{N}^{4} \mathrm{LO}\right)$ were derived by the Bochum-Bonn group [1,2]. Among important new features of these potentials, one should mention especially a new way of fixing values of some low energy constants (LECs) and a new regularization method. Namely, the values of LECs which occur in the long-range part of the interactions are taken directly from the pion-nucleon data without any fine tuning as it was done in older versions of the chiral NN potential. The second important change is the improved regularization scheme for the long-range part of the potential. The non-local regulator in momentum space, which was used in the previous versions, is now replaced by a local regulator in coordinate space. This allows one for a better control of regularization artifacts. In particular, such a procedure makes the long-rang part of the interaction less affected by the regularization procedure and removes unwanted short-range parts of the two-pion exchange potential. As a consequence, no spectral function regularization is needed.

The $2 \mathrm{~N}$ interactions of Ref. $[1,2]$ have been recently applied not only to $2 \mathrm{~N}$ scattering but also to the Nd scattering reactions [3]. This very first application proves usefulness of the chiral forces [1,2] in describing properties and processes in the $3 \mathrm{~N}$ systems. These results will be briefly reported in Section 2. In Section 3 we apply the same forces to radiative proton-deuteron capture. Also for this process new results are very promising. Especially, the weak dependence on the value of the regularization parameter increases the predictive power of the new forces compared to the older ones [4].

\footnotetext{
ae-mail: roman.skibinski@uj.edu.pl
} 


\section{Elastic neutron-deuteron scattering}

Elastic Nd scattering is the simplest process in $3 \mathrm{~N}$ systems in which the nuclear forces can be tested. Already for the cross section, strong $3 \mathrm{~N}$ force effects have been found at intermediate energies. However, a careful study of $3 \mathrm{NF}$ effects requires detailed knowledge of the $2 \mathrm{~N}$ interaction and a very weak cut-off dependence of theoretical predictions. To test the quality of the new chiral potentials, we calculate, neglecting the $3 \mathrm{~N}$ forces, the differential cross section for elastic $\mathrm{Nd}$ scattering. We solve in momentum space the Faddeev equation [5] for the state $T|\phi\rangle$,

$$
T|\phi\rangle=t P|\phi\rangle+t P G_{0} T|\phi\rangle
$$

with the off-the-energy shell $2 \mathrm{~N}$ t-matrix $t$, the sum $P$ of a cyclic and anti-cyclic permutation of three nucleons, the free $3 \mathrm{~N}$ propagator $G_{0}$, and the initial state $|\phi\rangle$, composed of the deuteron and a momentum eigenstate of the projectile nucleon. The $2 \mathrm{~N}$ interaction enters Eq. 1 via the t-matrix. The transition amplitude for elastic scattering with a nucleon-deuteron final state $\left|\phi^{\prime}\right\rangle$ is then given by [5]

$$
\left\langle\phi^{\prime}|U| \phi\right\rangle=\left\langle\phi^{\prime}\left|P G_{0}^{-1}\right| \phi\right\rangle+\left\langle\phi^{\prime}|P T| \phi\right\rangle .
$$

The first test of usefulness of the new model of the chiral force is the convergence of results with respect to the order of chiral expansion. This is shown in the left panel of Fig. 1 for the differential cross section at the relatively high nucleon laboratory energy of $E=200 \mathrm{MeV}$ and for the regularization parameter $R=0.9 \mathrm{fm}$. We clearly see, that going from next-to-leading order (NLO) to next-tonext-to-leading order $\left(N^{2} L O\right)$ and subsequently to $N^{3} L O$ causes a shift of predictions. The predictions at $N^{4} L O$ overlap with these at $N^{3} L O$ for most of the scattering angles. Only below $\theta \approx 20^{\circ}$, a tiny difference between $N^{3} L O$ and $N^{4} L O$ predictions is seen. Finally, we observe a nice agreement with predictions based on the AV18 $2 \mathrm{~N}$ force [6].

Next we check the cut-off dependence shown in Fig. 1 (the right panel) for the same energy and at $N^{4} L O$. That dependence of the cross section is very weak at this energy, however, it is stronger than at, not shown here, lower energies. The prediction obtained for $R=1.2 \mathrm{fm}$ lies further away from the other four curves what confirms the indication from $2 \mathrm{~N}$ scattering that such a value of the $R$ parameter generates a large amount of cut-off artifacts. While it is instructive to study cut-off dependence of the predictions, we want to stress that not all values of the cut-off parameter used here are equally preferred. In particular, the values of $R=0.9 \mathrm{fm}$ and $R=1.0 \mathrm{fm}$ lead to the $\chi^{2} /$ data ratio close to 1.0 for high energy $2 \mathrm{~N}$ scattering. It remains to check if the same values of the cut-off parameter will be preferred in $3 \mathrm{~N}$ reactions or nuclear structure calculations. We also want to emphasize that the cutoff dependence of predictions does not provide a proper way to estimate theoretical uncertainties in calculations of this kind. In Refs. [1,3] we formulated an approach to estimate truncation errors, that is the theoretical uncertainty due to neglected contributions from higher orders of the chiral expansion. This novel approach was justified for the case of $R=0.9 \mathrm{fm}$ in the Bayesian analysis of Ref. [7].

\section{The radiative proton-deuteron capture reaction}

The radiative pd capture reaction is another process to test the new chiral $2 \mathrm{~N}$ forces. Compared to elastic scattering, a new dynamical ingredient, namely the electromagnetic current operator $j_{\mu}$, enters the calculation. The transition amplitude for radiative capture can be obtained via time reversal symmetry from the transition amplitude $N_{\mu}^{N d}$ for two-body photodisintegration of the corresponding $3 \mathrm{~N}$ bound state, $\gamma+{ }^{3} \mathrm{He} \rightarrow p+d$ in our case $[8,9]$.

The corresponding Faddeev equation, if one neglects the $3 \mathrm{~N}$ interaction, is given as

$$
|U\rangle=t G_{0}(1+P) j_{\mu}\left|\Phi^{3 N}\right\rangle+t G_{0} P|U\rangle
$$



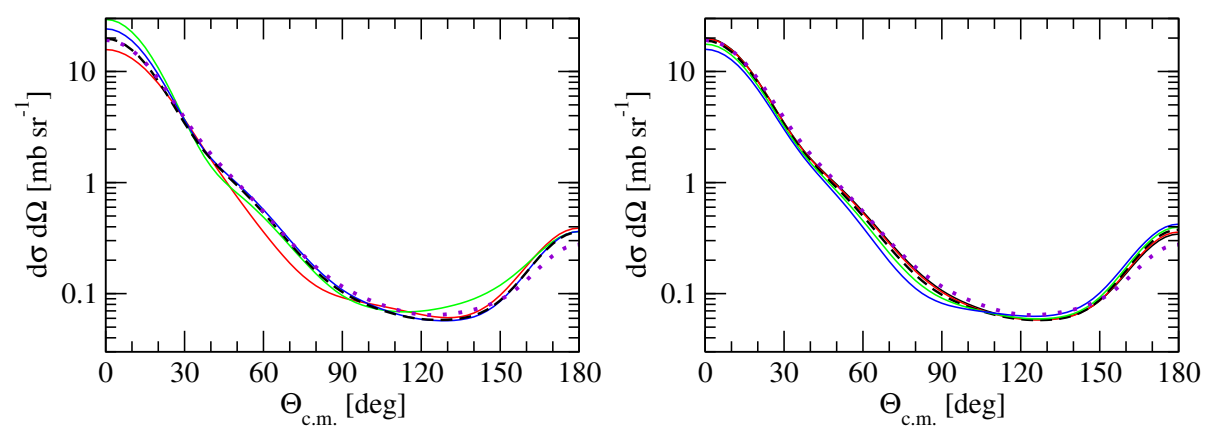

Figure 1. The neutron-deuteron elastic scattering differential cross section $d \sigma / d \Omega$ as a function of the center-ofmass angle $\theta_{c . m}$. at the neutron energy $E_{l a b}=200 \mathrm{MeV}$. On the left, the convergence of the cross section is shown for $R=0.9 \mathrm{fm}$. The red, green, blue and dashed black curves correspond to the predictions at $N L O, N^{2} L O, N^{3} L O$ and $N^{4} L O$, respectively. On the right, the cut-off dependence at $N^{4} L O$ is shown. The solid black, red, dashed black, green and blue curves correspond to the predictions with $R=0.8,0.9,1.0,1.1$ and $1.2 \mathrm{fm}$, respectively. In both panels the dotted violet line shows the AV18 predictions.

and the transition amplitude $N_{\mu}^{N d}$ can be calculated by

$$
N_{\mu}^{N d}=\left\langle\phi^{\prime}\left|(1+P) j_{\mu}\right| \Phi^{3 N}\right\rangle+\left\langle\phi^{\prime}|P| U\right\rangle,
$$

where the state $\left|\Phi^{3 N}\right\rangle$ is the ${ }^{3} \mathrm{He}$ wave function. In the following, we use the Siegert theorem to include implicitly many-body electromagnetic current operators (see $[8,9]$ for more details).

In Fig. 2 we show the differential cross section for proton-deuteron radiative capture at the incoming deuteron laboratory energy of $E=95 \mathrm{MeV}$. The left panel again shows the convergence with respect to the chiral order. While leading order predictions are far from others at forward and backward photon emission angles, all other curves are overlapping in this area. At the maximum of the cross section, the predictions at $N L O$ and $N^{2} L O$ come close together, however there is a shift in predictions when moving to $N^{3} L O$. The predictions at $N^{4} L O$ overlap with the $N^{3} L O$ ones. At this energy, the cut-off dependence is seen only in the region of the maximum of the cross section, see the right panel of Fig. 2). The cross section increases smoothly with the increasing value of the regularization parameter, however, the differences between the biggest and the lowest values of the cross section amount only to about $10 \%$, which is factor of two smaller than the corresponding spread of results for the older version of the chiral $2 \mathrm{~N}$ potential [10].

In summary, the improved chiral $2 \mathrm{~N}$ interactions of Refs. $[1,2]$ have been tested in elastic Nd scattering and in radiative pd capture. For both processes, the new forces show very promising behavior (reasonable values of observables, good convergence with respect to the chiral order and weak cut-off dependence). These findings are very promising in view of the ongoing work towards the inclusion of consistent chiral $3 \mathrm{~N}$ forces and electromagnetic currents.

\section{Acknowledgements}

This work was done as a part the LENPIC project and was supported from the resources of the National Science Center (Poland) under grant DEC-2013/10/M/ST2/00420. It was also supported in part by DFG (SFB/TR 16, "Subnuclear Structure of Matter") and ERC project 259218 NUCLEAREFT. Numerical calculations have been performed on the supercomputer clusters of the JSC, Jülich, Germany. 

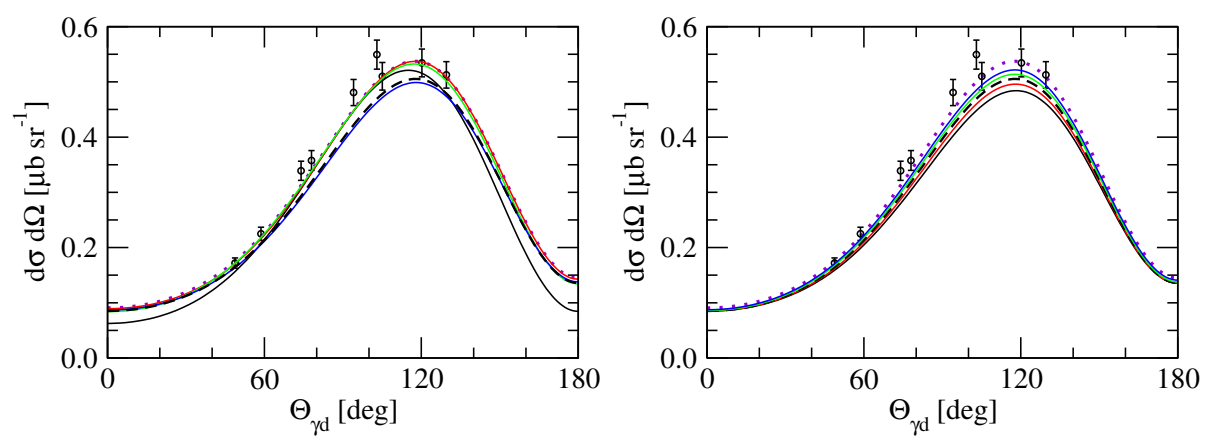

Figure 2. The differential cross section $d \sigma / d \Omega$ for proton-deuteron radiative capture as a function of the photon emission angle $\theta_{\gamma, d}$ at the deuteron laboratory energy $E_{l a b}=95 \mathrm{MeV}$. The panels and curves as in Fig.1, except for dotted curve which corresponds to predictions based on the AV18 and the UrbanaIX [11] interactions. Data are from [12].

\section{References}

[1] E. Epelbaum, H. Krebs, U.-G.Meißner, Eur. Phys. J. A5, 53 (2015).

[2] E. Epelbaum, H. Krebs, U.-G.Meißner, arXiv:1412.4623 [nucl-th] (2014).

[3] S.Binder et al., arXiv:1505.07218.

[4] H. Witała, J. Golak, R. Skibiński, K. Topolnicki, J. Phys. G: Nucl. Part. Phys. 41, 094011 (2014).

[5] W. Glöckle, H. Witała, D. Hüber, H. Kamada and J. Golak, Phys. Rep. 274, 107 (1996).

[6] R.B. Wiringa, V.G.J. Stoks, and R. Schiavilla, Phys. Rev. C 51, 38 (1995).

[7] R.J.Furnstahl, N.Klco, D.R.Phillips, S.Wesolowski, arXiv.1506.01343.

[8] J. Golak et al., Phys. Rev. C62, 054005 (2000).

[9] J. Golak et al., Phys. Rept. 415, 89 (2005)

[10] R.Skibiński et al. Acta Phys.Polon. B37 2905 (2006).

[11] B.S.Pudliner et al., Phys. Rev. C56, 1720 (1997).

[12] W.K. Pitts et al., Phys. Rev. C37, 1 (1988). 